\title{
Nanoscopy for the whole cell
}

Researchers plumb the depths of the cell with volumetric $4 \mathrm{Pi}$ nanoscopy.

Microscopes that enable whole-cell volumetric imaging in super-resolution would be very useful for studying the numerous subcellular structures that are smaller than $\sim 200 \mathrm{~nm}$, the diffraction-limited resolution of light microscopy. However, imaging beyond thin structures and deep into the entire volume of a cell with equally high resolution in all three dimensions, also known as isotropic resolution, has remained a challenge for super-resolution microscopy.

Joerg Bewersdorf at Yale University, along with colleagues including Fang Huang and George Sirinakis, two senior postdocs in his laboratory, sought to address this challenge and extend single-molecule localization microscopy to depths of 10 micrometers, enabling isotropic super-resolution imaging of structures throughout the entire cell.

Bewersdorf was inspired by his work in Stefan Hell's lab developing 4Pi microscopy, which he believes is the most efficient way to improve the depth resolution of a microscope. In 4Pi microscopy, which is a confocal approach, two opposing objective lenses are illuminated coherently and focus on the same spot. The confocal focus is sharpened by the interference of the counter propagating wavefronts, effectively increasing axial resolution approximately fourfold to sevenfold compared with conventional confocal microscopy.

This optical configuration has been used previously for 3D single-molecule localization microscopy. The most notable example is interferometric photoactivated localization microscopy (iPALM), developed by Harald Hess and colleagues at Janelia Research Campus. iPALM improves the axial resolution (relative to that of previous PALM instruments that use single objectives) to $10-20 \mathrm{~nm}$, yet the problem for this and related methods is "that applications had been limited to thin optical sections of about 1 micron at best and that applications were quite scarce," notes Bewersdorf.
To go far beyond 1 micrometer and image the entire depth of a cell grown on a coverslip, the researchers used a combination of specialized adaptive optics, carefully designed hardware for data collection and improved software algorithms for analysis. The adaptive optics were implemented with deformable mirrors to correct for optical aberrations that occur when imaging at depth. In terms of hardware, the optical setup was designed to maximize speed and stability. Finally, the data analysis algorithms enabled drift correction and stitching of optical sections to form the complete $3 \mathrm{D}$ data set. The team called their method whole-cell $4 \mathrm{Pi}$ single-molecule switching nanoscopy (W-4PiSMSN).

To demonstrate the performance of $\mathrm{W}-4 \mathrm{PiSMSN}$, the team imaged a wide range of samples and structures. First, they examined the endoplasmic reticulum in mammalian cells. They were able to image the network of 60-100-nm tubes in superresolution and resolve the $3 \mathrm{D}$ membrane contour, which otherwise could be resolved only with electron tomography. Next, they labeled microtubules, which allowed them to determine that the resolution of their system in all three dimensions was well below $20 \mathrm{~nm}$.

Another challenging target that has previously been imaged only by electron microscopy is the T7 bacteriophage. These viruses are known to feature an icosahedralshaped capsid that is $\sim 60 \mathrm{~nm}$ in diameter. Images taken with W-4PiSMSN allowed the researchers to see the hexagonal and pentagonal geometry of the phages despite their minute size, which surprised Bewersdorf, who recalls, "I knew our microscope was good, but not that good!"

The team also demonstrated multicolor, volumetric super-resolution imaging of microtubules and mitochondria; whole-cell imaging of mitochondria; whole-nucleus imaging of nuclear pore complexes; and 3D imaging of COPI vesicles associated with the Golgi apparatus. They further demonstrated that their approach could enable visualization of synaptonemal complexes in mouse

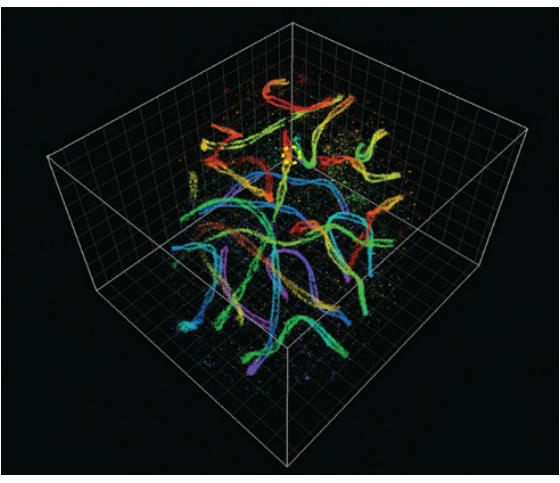

Synaptonemal complexes in whole-mouse spermatocytes imaged with W-4PiSMSN. Figure adapted from Huang et al. (2016) with permission (https://creativecommons.org/ licenses/by/4.0/).

spermatocyte nuclei, where they were able to image the twisting band of paired lateral elements of synaptonemal complexes with unprecedented clarity, setting the stage for better understanding of the chromosomal scaffolds in their native context.

$\mathrm{W}-4 \mathrm{PiSMSN}$ is already quite sophisticated, but Bewersdorf and his team see room for improvement. One drawback is the image acquisition time, which ranged from 5 minutes to an hour in their work, depending on the sample. This limits the method to fixed cells, although the researchers are developing improvements that could potentially increase the speed enough to enable live-cell imaging. The team is also working on improving the adaptive optics and illumination in order to image even thicker samples.

To disseminate this technology, Bewersdorf and his team are currently helping four labs around the world build their own W-4PiSMSN microscopes, and they are working with collaborators to address biological questions. This and future work promise an exciting future for volumetric 3D super-resolution microscopy.

\section{Rita Strack}

\section{RESEARCH PAPERS}

Huang, F. et al. Ultra-high resolution 3D imaging of whole cells. Cell 166, 1028-1040 (2016). 